\title{
The Use of Clozapine in the Serious Mental Illness Patients Enrolled in an Assertive Community Treatment Program
}

Maria Ruiza Yee 1, 2, 3 , Eduardo Espiridon ${ }^{4}$, Adeolu O. Oladunjoye ${ }^{5,3}$, Udema Millsaps ${ }^{3}$, Nailah Harvey ${ }^{2}$, Anish H. Vora ${ }^{1}$

1. Psychiatry, Drexel University College of Medicine, Philadelphia, USA 2. Psychiatry, Philadelphia College of Osteopathic Medicine, Philadelphia, USA 3. Psychiatry, Reading Hospital Tower Health, West Reading, USA 4. Psychiatry, Tower Health Medical Group, West Reading, USA 5. Medical Critical Care, Boston Children's Hospital, Boston, USA

Corresponding author: Eduardo Espiridon, edjen19meg@gmail.com

\section{Abstract}

\section{Introduction}

Health care cost is projected to reach 20\% of the nation's gross national product (GNP) by $2016.6 .2 \%$ of this is from mental health. The National Institute of Mental Health (NIMH) estimates the prevalence of serious mental illness (SMI) at 13.1 million or $5.2 \%$ of American adults age 18 or over. Hence, mental health care cost for this patient population is significant.

Patients with SMI involved in an Assertive Community Treatment (ACT) program are individuals who experience the most intractable symptoms and the greatest level of dysfunction from their mental illness. These individuals typically are institutionalized in a long-term facility such as the state hospital.

Clozapine has shown superior efficacy over first- and most second-generation antipsychotics in both treating treatment-resistant and non-treatment-resistant schizophrenia which has been supported by several large trials. There is also evidence of its efficacy in suicidality, aggression and substance misuse. In fact, clozapine has been approved by the FDA for use in refractory schizophrenia and suicidality in schizoaffective disorder. Due to the risk of agranulocytosis, clozapine is underutilized. The purpose of this study is to conduct a retrospective cohort study through chart review to analyze whether the addition of clozapine to ACT treatment of SMI patients in a community hospital from 2008 to 2018 led to decreased frequency of hospitalizations and increased clinical stability.

\section{Materials and methods}

A retrospective study using electronic medical record (EMR) of patients ages 20 and above who were enrolled in the ACT program at a community hospital from December 1, 2008 to December 31, 2018. Variables were collected from the EMR and de-identified during data collation. Analysis was performed using SPSS software package.

Review began 05/12/2021 Review ended 05/18/2021 Published 05/25/2021

\section{() Copyright 2021}

Yee et al. This is an open access article distributed under the terms of the Creative Commons Attribution License CC-BY 4.0., which permits unrestricted use, distribution, and reproduction in any medium, provided the original author and source are credited.

\section{Results}

A total of 179 patients enrolled in the ACT program and their data was extracted from the EMR. Twenty-five (62.5\%) of these patient enrollments were on clozapine. They were made up of 53.6\% male, $81.9 \%$ White/Asian, $18.1 \%$ Black; $44.1 \%$ ages between 36 to 50 years old, $30.2 \%$ were aged 18 to 35 years old, and $25.7 \%$ greater than 50 years old. There was no difference in age, sex, race, ethnicity, and insurance type in ACT program between those using clozapine and those not on clozapine. There was a higher proportion of psychiatric hospitalizations among clozapine users compared with the non-clozapine user group ( $62.5 \% \mathrm{vs}$ $41.5 \%, \mathrm{p}=0.019$ ). However, the two groups did not differ from one another in terms of psychiatric emergency visits $(p=0.128)$ or frequency of ACT visits $(p=0.002)$.

\section{Conclusion}

Effective treatment that will reduce hospitalizations and the burden of chronic disability in patients with SMI would greatly reduce mental health care cost. Clozapine remains the gold standard in the treatment of refractory schizophrenia. But due to the risk of agranulocytosis, clozapine is underutilized. It was hoped that this study will support the use of clozapine in SMI patients. Disappointingly, the use of clozapine did not prevent relapses and hospitalizations in this patient population and patients on clozapine seemed to have increased hospitalizations, compared to those who were not on clozapine. Perhaps, a different outcome would have occurred if the focus was limited to the patients themselves who were on Clozapine and ascertain what the rate of hospitalization was before the start of clozapine vs after the use of clozapine. 
Categories: Psychiatry, Public Health

Keywords: smi, act, clozapine, health care cost, community hospital

\section{Introduction}

Patients with serious mental illness (SMI) pose a significant mental health cost, not only due to the cost of acute care from inpatient psychiatric hospitalizations and frequent emergency room visits but also from indirect cost from loss of income from unemployment, the social services required to support these individuals in their chronic illness and various other indirect costs to support the chronic disability.

Serious mental illness, as defined by the National Institute of Mental Health (NIMH) is a mental, behavioral or emotional disorder resulting in serious functional impairment which substantially interferes with or limits one or more major life activities [1]. SMI is made up of a smaller group of mental health illnesses and a more severe type of mental illness [2]. NIMH reports that approximately 13.1 million or $5.2 \%$ of American adults age 18 or over, as of 2019 have SMI [1], with the highest prevalence in young adults, ages 18 to 25 [1]. There is a higher risk in females as compared to males. Economically, SMI was estimated to cost the health care sector over $20 \%$ of the nations' gross national domestic product (GDP) in 2016 [3]. Mental health care constitutes $6.2 \%$ of health care costs in the United States. The mean reduction in earnings in persons with SMI is $\$ 16,306$ [3]. Hence, it is important to prevent relapses and ensure clinical stability in this patient population.

Patients with SMI experience highly intractable symptoms and great levels of dysfunction from their mental illness [2]. Patients who suffer from SMI do not respond to standard outpatient psychiatric care and will need an alternative that is effective and efficient. Assertive Community Treatment (ACT) program is an effective [4-6], and cost-effective treatment [7-9] in this group of individuals, especially in those with extensive prior hospital utilization. ACT program not only results in a reduction of psychiatric hospitalization but also, more stable socioeconomic condition [2]. Patients with SMI involved in an ACT program are individuals who experience the most intractable symptoms and the greatest level of dysfunction from their mental illness.

ACT program was started over 30 years ago by a group of mental health professionals at the Mendota Mental Health Institute in Wisconsin [5]. It started because of the high rate of recidivism in individuals with SMI who were discharged in stable conditions. They designed a service delivery model that is now recognized as the ACT program. A typical team membership includes 10 to 12 professionals from the different fields in mental health including fields of psychiatry, psychology, and other allied health care professionals who provided services needed by these patients [5]. Services are provided every time of the day and every day of the week [2]. Patients are taught life skills for real-life situations as well[2].

Prevention of relapse is very essential in SMI as psychopathology, social and occupational functioning worsen with repeated psychotic episodes [2]. Relapse can also lead to higher healthcare costs and hence an economic burden on a nation. Clozapine has shown superior efficacy than first- and most second-generation antipsychotics in both treating treatment-resistant and non-treatment-resistant schizophrenia which has been supported by several large trials [10-12]. There is also evidence of its efficacy in suicidality, aggression and substance misuse [12]. In fact, clozapine has been approved by the FDA for use in refractory schizophrenia and suicidality in schizoaffective disorder. Due to the risk of agranulocytosis, clozapine is underutilized [13]. The purpose of this study is to ascertain whether the addition of clozapine to ACT treatment of SMI patients led to a decrease in the frequency of hospitalizations and improvement in clinical stability.

\section{Materials And Methods}

This was a retrospective study conducted using data from the electronic medical record (EMR). The patient population studied included patients ages 20 and above who were enrolled at an outpatient ACT program at a community hospital from December 1, 2008 to December 31, 2018. Their data included diagnoses using the International Classification of Diseases (ICD-9) and ICD-10 codes. The ICD-10 diagnostic codes extracted were Manic episode F 30.x; Bipolar disorder F 31.x Schizophrenia F 20.x, and Schizoaffective disorder F 25.x. While the ICD-9 codes extracted were Schizophrenic disorders 295.x; Episodic mood disorders 296.x and Delusional disorders 297.x. Data on medications given during the study period to these patients were also extracted from the EMR. These included oral antipsychotic medications, both typical and atypical, and other medications administered during patient care in the ACT program.

A total of 189 patient enrollments in the ACT program were included in data collection. There were five patients who dropped out of the ACT program and five patients with incomplete/inappropriate data information which were excluded from the final analysis. Of the 179 patients remaining in the analysis, eight patients had two separate encounters in the ACT program while 163 patients had only one encounter in the ACT program. Patient hospitalizations were divided into two arms, i.e., those who received clozapine and those who did not receive clozapine.

To ascertain if clozapine was effective in reducing the number of hospitalizations, the number of hospitalizations was recorded after the start of clozapine versus the other arm where hospitalizations were 
recorded in those who were not on clozapine. Variables collected from the EMR were de-identified into an Excel spreadsheet for data collation and analysis was performed using SPSS software (IBM Corp., Armonk, $\mathrm{NY})$ package.

\section{Statistical analysis}

In our statistical analysis, we described the prevalence of clozapine among SMI patients. Summary statistics were presented using proportions, mean and standard deviation. The mean number of hospitalizations, psychiatric emergency visits and number of planned ACT visits in the two groups (clozapine vs nonclozapine treatment) were compared.

Multivariate linear regression analysis was used to adjust for other independent co-variables. Kaplan Meier analysis was used to compare time from ACT enrollment to the first hospitalization. The level of significance was set at $\mathrm{p}<0.05$. All statistical analyses were performed using SPSS software package.

\section{Results}

In our study, a total of 179 enrolments were analyzed. In 15 (37.5\%) enrolments, clozapine was used. The enrolments were made up of 53.6\% Male, 81.9\% White or Asian, 18.1\% Black, 44.1\% ages between 36 and 50 years old. The overall mean age was $42.5 \pm 11.8$ years (Table 1 ). Table 1 describes the demographic and clinical characteristics of patients with SMI enrolled at the ACT program who were placed on clozapine. Insurance coverage varied with Medicaid insurance (56.5\%) being the predominant insurance coverage, followed by Medicare insurance (36.2\%), and private insurance or workman's compensation (7.3\%). Most of the patients were unemployed or retired (95.9\%). The most common diagnosis was schizoaffective disorder (44.0\%), with schizophrenia (35.4\%), bipolar disorder (16.0\%) and major depressive disorder (4.6\%) coming thereafter. Co-morbid substance abuse was significant with alcohol being the most commonly abused at $54.2 \%$, followed by marijuana at $44.1 \%$ and cocaine at $29.6 \%$. Most of the patients were enrolled in the ACT program for an average of one to five years (46.7\%).

\begin{tabular}{|c|c|c|c|c|}
\hline Name & All SMI $(n=179)$ & Clozapine use $(n=40)$ & No clozapine use $(n=139)$ & p-value \\
\hline Mean age $( \pm \mathrm{SE})$ & $42.5 \pm 11.8$ & $39.9 \pm 10.9$ & $43.3 \pm 12.0$ & \\
\hline \multicolumn{5}{|l|}{ Age, years } \\
\hline 18-35 & $54(30.2 \%)$ & $16(40.0 \%)$ & $38(27.3 \%)$ & \\
\hline $36-50$ & 79 (44.1\%) & 15 (37.5\%) & $64(46.0 \%)$ & \\
\hline$>50$ & $46(25.7 \%)$ & $9(22.5 \%)$ & $37(26.6 \%)$ & 0.306 \\
\hline \multicolumn{5}{|l|}{ Sex } \\
\hline Female & $83(46.4 \%)$ & $18(45.0 \%)$ & 65 (46.8\%) & \\
\hline Male & $96(53.6 \%)$ & $22(55.0 \%)$ & $74(78.3 \%)$ & 0.844 \\
\hline \multicolumn{5}{|l|}{ Race } \\
\hline White/Asian & 145 (81.9\%) & $35(89.7 \%)$ & $110(79.7 \%)$ & \\
\hline Black & $32(18.1 \%)$ & $4(10.3 \%)$ & $28(20.3 \%)$ & 0.151 \\
\hline \multicolumn{5}{|l|}{ Ethnicity } \\
\hline Hispanic & $44(24.6 \%)$ & $10(25.0 \%)$ & $34(24.5 \%)$ & \\
\hline Non-Hispanic & $135(75.4 \%)$ & $30(75.0 \%)$ & $28(75.5 \%)$ & 0.944 \\
\hline \multicolumn{5}{|l|}{ Insurance } \\
\hline Medicaid & $100(56.5 \%)$ & $23(57.5 \%)$ & 77 (56.2\%) & \\
\hline Medicare & $64(36.2 \%)$ & $15(37.5 \%)$ & 49 (35.8\%) & \\
\hline Private/ worker's compensation & $13(7.3 \%)$ & $2(5.0 \%)$ & $11(8.0 \%)$ & 0.810 \\
\hline \multicolumn{5}{|l|}{ Employment } \\
\hline Unemployed/retired & $162(95.9 \%)$ & 38 (97.4\%) & 124 (95.4\%) & \\
\hline Employed/self-employed & 7 (4.1\%) & 1 (2.6\%) & 6 (4.6\%) & 1.000 \\
\hline
\end{tabular}




\section{Cureus}

Bipolar disorders
Major depressive disorder
Schizoaffective disorders
Schizophrenia

Substance use disorder

\section{No}

Yes

Marijuana

Cocaine

Alcohol

Smoking

No

Yes

Days in ACT program

$<1$ year

1-5 years

$>5$ years

Number of psychiatric hospitalizations

$$
\begin{aligned}
& 0 \text { No visit } \\
& \geq 1 \text { visit }
\end{aligned}
$$

Number of psychiatric emergency visits

$$
\begin{aligned}
& 0 \text { No visit } \\
& \geq 1 \text { visit }
\end{aligned}
$$

Planned ACT visits

\section{$<100$ visits}

100-500 visits

$>500$ visits

$\begin{array}{ll}28(16.0 \%) & 3(7.5 \%) \\ 8(4.6 \%) & 1(2.5 \%) \\ 77(44.0 \%) & 19(47.5 \%) \\ 62(35.4 \%) & 17(42.5 \%)\end{array}$

$25(18.5 \%)$

$7(5.2 \%)$

$58(43.0 \%)$

$45(33.3 \%)$

0.295

$51(28.5 \%) \quad 15(37.5 \%) \quad 36(25.9 \%)$

$128(71.5 \%) \quad 25(62.5 \%)$

$103(74.1 \%)$

0.152

$59(42.4 \%)$

0.397

$40(28.8 \%)$

0.649

$77(55.4 \%)$

0.546

$31(22.3 \%)$

$99(71.2 \%)$

0.138

$126(70.4 \%)$

$27(67.5 \%)$

$\begin{array}{ll}58(42.3 \%) & 7(30.4 \%) \\ 64(46.7 \%) & 10(43.5 \%) \\ 15(10.9 \%) & 6(26.1 \%)\end{array}$

$51(44.7 \%)$

$54(47.4 \%)$

9 (7.9\%)

0.034

$\begin{array}{ll}94(53.7 \%) & 15(37.5 \%)\end{array}$

79 (58.5\%)

56 (41.5\%)

0.019

$109(64.1 \%)$

$21(53.8 \%)$

$88(67.2 \%)$

$61(35.9 \%)$

$18(46.2 \%)$

$43(32.8 \%)$

0.128

$\begin{array}{lll}36(20.6 \%) & 5(12.8 \%) & 31(22.8 \%) \\ 82(46.9 \%) & 12(30.8 \%) & 70(51.5 \%) \\ 57(32.6 \%) & 22(56.4 \%) & 35(25.7 \%)\end{array}$

TABLE 1: Baseline and clinical characteristics of patients with serious mental illness (SMI) enrolled at the Assertive Community Treatment (ACT) program with the use of clozapine and without the use of clozapine.

$\mathrm{n}=$ sample number; SE: Standard error; \%: percentage.

There was no difference in age, sex, race, ethnicity, and insurance type between those using clozapine and those not on clozapine. Most patients spent an average of 1-5 years in the ACT program (46.7\%). When compared with the no clozapine group, a higher proportion of those in the clozapine group spent $>5$ years in the ACT program $(26.1 \%$ vs $7.9 \%, p=0.034)$ and had at least one psychiatric hospitalization $(62.5 \%$ vs $41.5 \%$, $\mathrm{p}=0.019)$. Of those who used clozapine, $46.2 \%(18 / 40)$ had at least one psychiatric emergency visit, whereas among those who did not use clozapine, 32.8\% (43/139) had at least one psychiatric emergency visit. However, this was not statistically significant. The majority of planned ACT visits were between 100 and 500 visits during their program participation (46.9\%).

\section{Association of psychiatric hospitalizations with clozapine use}




\section{Cureus}

Univariate analysis showed that clozapine use was associated with psychiatric hospitalizations (OR 2.30, $95 \%$ CI 1.25-4.24, $\mathrm{p}=0.021$ ). Males were less likely to have psychiatric hospitalizations (OR 0.47, CI 0.26$0.87, \mathrm{p}=0.015$ ). Those with substance use disorders were less likely to have psychiatric hospitalizations compared to those who did not have substance use disorder (OR 0.46, 95\% CI 0.24-0.90, p = 0.023) (Table 2).

\begin{tabular}{|c|c|c|c|c|}
\hline Name & Univariate analysis (crude OR) & p-value & Multivariate analysis (adjusted OR) ${ }^{\ddagger}$ & p-value \\
\hline Clozapine use & $2.30(1.25-4.24)$ & $0.021^{*}$ & $2.41(0.86-6.77)$ & 0.960 \\
\hline \multicolumn{5}{|l|}{ Age, years } \\
\hline 18-35 & Ref & & & \\
\hline $36-50$ & $0.96(0.48-1.93)$ & 0.909 & & \\
\hline$>50$ & $0.93(0.42-2.08)$ & 0.866 & & \\
\hline \multicolumn{5}{|l|}{ Sex } \\
\hline Female & Ref & & & \\
\hline Male & $0.47(0.26-0.87)$ & $0.015^{\star}$ & $0.26(0.12-0.55)$ & $<0.0001$ \\
\hline \multicolumn{5}{|l|}{ Race } \\
\hline White/Asian & Ref & & & \\
\hline Black & $0.81(0.37-1.77)$ & 0.596 & & \\
\hline \multicolumn{5}{|l|}{ Ethnicity } \\
\hline Hispanic & Ref & & & \\
\hline Non-Hispanic & $0.64(0.32-1.28)$ & 0.206 & & \\
\hline \multicolumn{5}{|l|}{ Insurance } \\
\hline Medicaid & Ref & & & \\
\hline Medicare & $0.99(0.52-1.86)$ & 0.964 & & \\
\hline Private/worker's compensation & $0.99(0.31-3.16)$ & 0.987 & & \\
\hline \multicolumn{5}{|l|}{ Employment } \\
\hline Unemployed/retired & Ref & & & \\
\hline Employed/self-employed & $0.43(0.08-2.29)$ & 0.324 & & \\
\hline \multicolumn{5}{|l|}{ Diagnosis } \\
\hline Bipolar disorders & Ref & & & \\
\hline Major depressive disorder & $0.60(0.12-3.01)$ & 0.534 & & \\
\hline Schizoaffective disorders & $0.90(0.38-2.14)$ & 0.807 & & \\
\hline Schizophrenia & $0.85(0.35-2.08)$ & 0.719 & & \\
\hline \multicolumn{5}{|l|}{ Substance use disorder } \\
\hline No & Ref & & & \\
\hline Yes & $0.46(0.24-0.90)$ & $0.023^{*}$ & $0.89(0.39-2.03$ & 0.785 \\
\hline Marijuana & $0.82(0.45-1.50)$ & 0.521 & & \\
\hline Cocaine & $0.64(0.33-1.23)$ & 0.179 & & \\
\hline Alcohol & $0.63(0.35-1.15)$ & 0.131 & & \\
\hline \multicolumn{5}{|l|}{ Smoking } \\
\hline No & Ref & & & \\
\hline Yes & $0.97(0.48-1.94)$ & 0.922 & & \\
\hline
\end{tabular}




\section{Cureus}

Years in ACT program

$<1$ year Ref

1-5 years

$1.90(0.91-3.96)$

$0.088 \quad 1.80(0.82-3.94)$

0.143

$>5$ years

Planned ACT outpatient visits

$<100$ visits

Ref

$100-500$ visits

$1.63(0.71-3.74)$

0.253

$>500$ visits

$1.81(0.75-4.37)$

0.185

TABLE 2: Association of psychiatric hospitalizations with clozapine use in serious mental illness (SMI) at the Assertive Community Treatment (ACT) program.

*Univariate statistical significance; $¥$ accommodated univariate statistical significance of 0.1 . Ref: Reference category used as analysis comparison.

However, after adjusting for other covariates, there was no association between clozapine use and psychiatric hospitalization (OR 2.41, 95\% CI $0.86-6.77, \mathrm{p}=0.960$ ). Sex remained an independent risk factor for psychiatric hospitalization with males being less likely to be hospitalized (OR 0.24, 95\% CI 0.11-0.52, p < 0.0001) (Table 2).

The mean time to first psychiatric hospitalization was 5.14 years for those on clozapine while it was 8.06 years for those not using clozapine $(\mathrm{p}=0.008)$. At 1.638 years, $50 \%$ of those on clozapine and $33 \%$ of those not on clozapine had experienced a psychiatric hospitalization. The probability of psychiatric hospitalization at one and five years was higher in the clozapine group (45\% and 59\%, respectively) than in the no clozapine group (28\% and $38 \%$, respectively) $(\mathrm{p}=0.008)$ (Figure 1$)$.

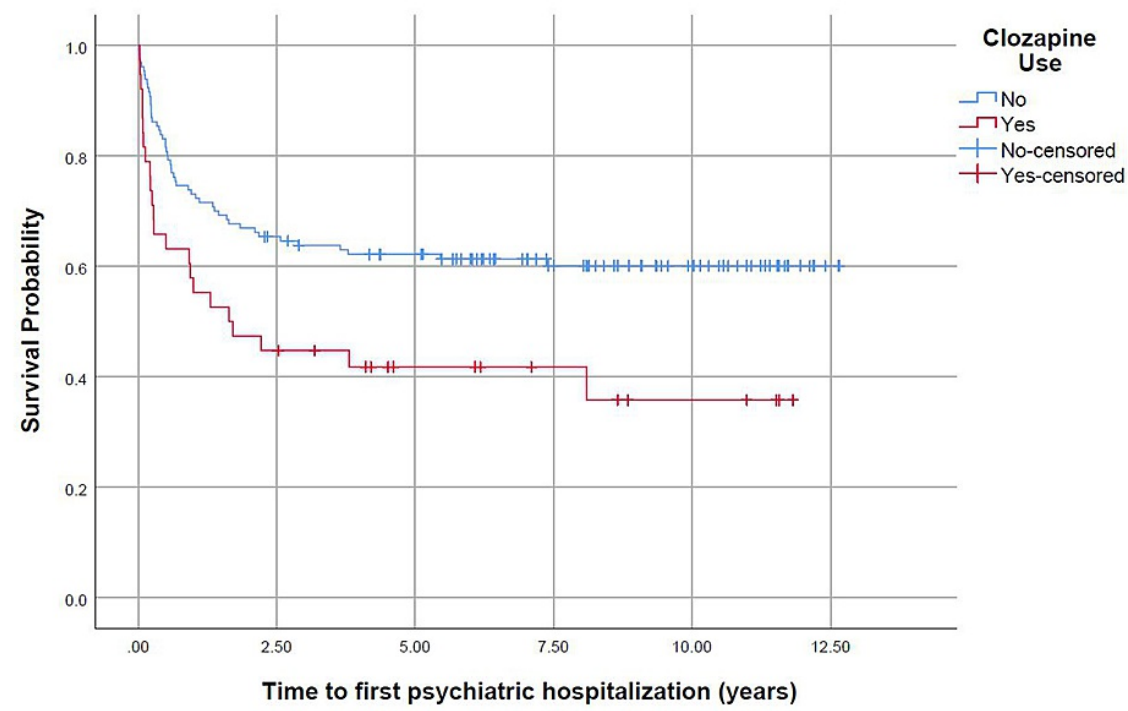

FIGURE 1: Plot comparing the probability of psychiatric hospitalization by clozapine use.

Kaplan Meier curve analysis.

\section{Discussion}

Patients with SMI typically do not respond to standard outpatient psychiatric care [2]. Hence the introduction of ACT for SMI has made a remarkable difference in patients in the program. ACT has proven to be not only an effective [4-6] but also a cost-effective treatment [7-9] especially in those with extensive prior hospital use. The ACT program does not only lead to the reduction of psychiatric hospitalization but also, in 
Clozapine has shown superior efficacy over first- and most second-generation antipsychotics in both treating treatment-resistant and non-treatment-resistant schizophrenia which has been supported by several large trials [10-12]. There is also evidence of its efficacy in suicidality, aggression and substance misuse [12]. In fact, clozapine has been approved by the FDA for use in refractory schizophrenia and suicidality in schizoaffective disorder. The patient outcome research team (PORT) initiated by the US Department of Health and Human Services recommends the use of clozapine in refractory schizophrenia after at least two adequate trials including at least one second-generation antipsychotic medication [13].

Due to the risk of agranulocytosis, clozapine is underutilized [14]. Other troublesome side effects such as excessive salivation, constipation, weight gain, seizure and rare significant risk of cardiomyopathy make it challenging for some patients and providers to use $[15,16]$. While clozapine remains underutilized, it has been shown to have benefits in reducing both positive and negative symptoms, and it is cost-effective in the long-term reduction in hospital admissions [17,18]. It has been reported, though, that many prescribers may, instead of transitioning to clozapine, prefer to use higher than PORT-recommended doses of other antipsychotics thereby delaying the use of clozapine in their patients [15,19]. Surprisingly, the results of our study do not substantiate the efficacy of clozapine in this patient population. This is despite the high level of adherence to clozapine prescribed while they were in the ACT program. If anything, it was associated with increased hospitalizations which is different from what is reported in previous studies.

It is worthwhile mentioning that the higher proportion of those on clozapine spent $>5$ years in the ACT program compared to those not on clozapine which was statistically significant $p=0.034$ and those on clozapine had a higher proportion of patients with over 500 visits during their participation in the ACT program which was also statistically significant $\mathrm{p}=0.002$. Hence, this may indicate that this patient population is a more refractory subset of patients with SMI.

\section{Conclusions}

Health care cost is one of the most daunting challenges of public policy in the United States. Hence, effective treatment that will reduce hospitalizations and the burden of chronic disability in patients with SMI would greatly reduce mental health care cost. Clozapine remains the gold standard in the treatment of refractory schizophrenia. But due to the risk of agranulocytosis, clozapine is underutilized. It was hoped that this study will support the use of clozapine in SMI patients. Disappointingly, the use of clozapine did not prevent relapses and hospitalizations in this patient population and in fact, patients on clozapine seemed to have increased hospitalizations, compared to those who were not on clozapine. It would seem that the use of clozapine was not contributory in maintaining clinical stability in this patient population. Perhaps, this is an indication that SMI patients in an ACT program constitute a very severe patient population and is not responsive to the use of clozapine. Perhaps, a different outcome would have occurred if the focus was limited to the patients themselves who were on Clozapine and ascertain what the rate of hospitalization was before the start of clozapine vs after the use of clozapine.

\section{Additional Information}

\section{Disclosures}

Human subjects: All authors have confirmed that this study did not involve human participants or tissue. Animal subjects: All authors have confirmed that this study did not involve animal subjects or tissue. Conflicts of interest: In compliance with the ICMJE uniform disclosure form, all authors declare the following: Payment/services info: All authors have declared that no financial support was received from any organization for the submitted work. Financial relationships: All authors have declared that they have no financial relationships at present or within the previous three years with any organizations that might have an interest in the submitted work. Other relationships: All authors have declared that there are no other relationships or activities that could appear to have influenced the submitted work.

\section{References}

1. National Institute of Mental Health. Mental illness . (2021). Accessed: April 14, 2021 : https://www.nimh.nih.gov.

2. Yee MR, Espiridon E, Oladunjoye AO, Millsaps U, Vora A, Harvey N: The use of long-acting injectable antipsychotics (LAI) in the serious mental illness (SMI) patients enrolled in an assertive community treatment (ACT) program. Cureus. 2021, 13:e14490. 10.7759/cureus.14490

3. Insel TR: Assessing the economic costs of serious mental illness. Am J Psychiatry. 2008, 165:663-5. 10.1176/appi.ajp.2008.08030366

4. Udechuku A, Olver J, Hallam K, et al.: Assertive community treatment of the mentally ill: service model and effectiveness. Australas Psychiatry. 2005, 13:129-34. 10.1080/j.1440-1665.2005.02175.x

5. Phillips SD, Burns BJ, Edgar ER, et al.: Moving assertive community treatment into standard practice . Psychiatr Serv. 2001, 52:771-9. 10.1176/appi.ps.52.6.771

6. Burns BJ, Santos AB: Assertive community treatment: an update of randomized trials . Psychiatr Serv. 1995, 46:669-75. 10.1176/ps.46.7.669

7. Bond GR, Drake RE, Mueser KT, et al.: Assertive community treatment for people with severe mental illness . 
Dis Manage Health Outcomes. 2001, 9:141-59. 10.2165/00115677-200109030-00003

8. Latimer EA: Economic impacts of assertive community treatment: a review of the literature . Can J Psychiatry. 1999, 44:443-54. 10.1177/070674379904400504

9. Rosenheck R, Neale M: Intersite variation in the impact of intensive psychiatric community care on hospital use. Am J Orthopsychiatry. 1998, 68:191-200. 10.1037/h0080329

10. Lewis SW, Barnes TR, Davies L, et al.: Randomized controlled trial of effect of prescription of clozapine versus other second-generation antipsychotic drugs in resistant schizophrenia. Schizophr Bull. 2006, 32:715-23. 10.1093/schbul/sbj067

11. McEvoy JP, Lieberman JA, Stroup TS, et al.: Effectiveness of clozapine versus olanzapine, quetiapine, risperidone in patients with chronic schizophrenia who did not respond to prior atypical antipsychotic treatment . Am J Psychiatry. 2006, 163:600-10. 10.1176/appi.ajp.163.4.600

12. Farooq S, Taylor M: Clozapine: dangerous orphan or neglected friend? . Br J Psychiatry. 2011, 198:247-9. 10.1192/bjp.bp.110.88690

13. Lehman AF, Kreyenbuhl J, Buchanan RW, et al.: The Schizophrenia Patient Outcomes Research Team (PORT): updated treatment recommendations 2003. Schizophr Bull. 2004, 30:193-217.

10.1093/oxfordjournals.schbul.a007071

14. Kane J, Honigfeld G, Singer J, Meltzer H: Clozapine for the treatment-resistant schizophrenic. A doubleblind comparison with chlorpromazine. Arch Gen Psychiatry. 1988, 45:789-96. 10.1001/archpsyc.1988.01800330013001

15. Hermes E, Rosenheck R: Choice of randomization to clozapine versus other second generation antipsychotics in the CATIE schizophrenia trial. J Psychopharmacol. 2012, 26:1194-200. $10.1177 / 0269881112443744$

16. Young CR, Bowers MB Jr, Mazure CM: Management of the adverse effects of clozapine . Schizophr Bull. 1998, 24:381-90. 10.1093/oxfordjournals.schbul.a033333

17. Rosenheck R, Cramer J, Xu W, et al.: A comparison of clozapine and haloperidol in hospitalized patients with refractory schizophrenia. Department of Veterans Affairs Cooperative Study Group on Clozapine in Refractory Schizophrenia. N Engl J Med. 1997, 337:809-15. 10.1056/NEJM199709183371202

18. Percudani M, Fattore G, Galletta J, Mita PL, Contini A, Altamura AC: Health care costs of therapy-refractory schizophrenic patients treated with clozapine: a study in a community psychiatric service in Italy. Acta Psychiatr Scand. 1999, 99:274-80. 10.1111/j.1600-0447.1999.tb07225.x

19. Sernyak MJ, Rosenheck R: Clinicians' reasons for deviations from recommended dosing practices for antipsychotic medications. Adm Policy Ment Health. 2007, 34:540-7. 10.1007/s10488-007-0142-y 